\title{
Plutarque chez Montaigne et chez Shakespeare1 janvier 2007
}

\section{Gisèle Mathieu-Castellani}

\author{
(2) OpenEdition \\ Journals \\ Édition électronique \\ URL : http://journals.openedition.org/shakespeare/671 \\ DOI : $10.4000 /$ shakespeare. 671 \\ ISSN : 2271-6424 \\ Éditeur \\ Société Française Shakespeare \\ Édition imprimée \\ Date de publication : 1 novembre 2004 \\ Pagination : 195-208 \\ ISBN : 2-9521475-0-7
}

\section{Référence électronique}

Gisèle Mathieu-Castellani, «Plutarque chez Montaigne et chez Shakespeare1 janvier 2007 », Actes des congrès de la Société française Shakespeare [En ligne], 21 | 2004, mis en ligne le 01 novembre 2003, consulté le 03 mai 2019. URL : http://journals.openedition.org/shakespeare/671 ; DOI : 10.4000/ shakespeare.671 


\section{Shakespeare et Montaigne vers un nouvel humanisme}

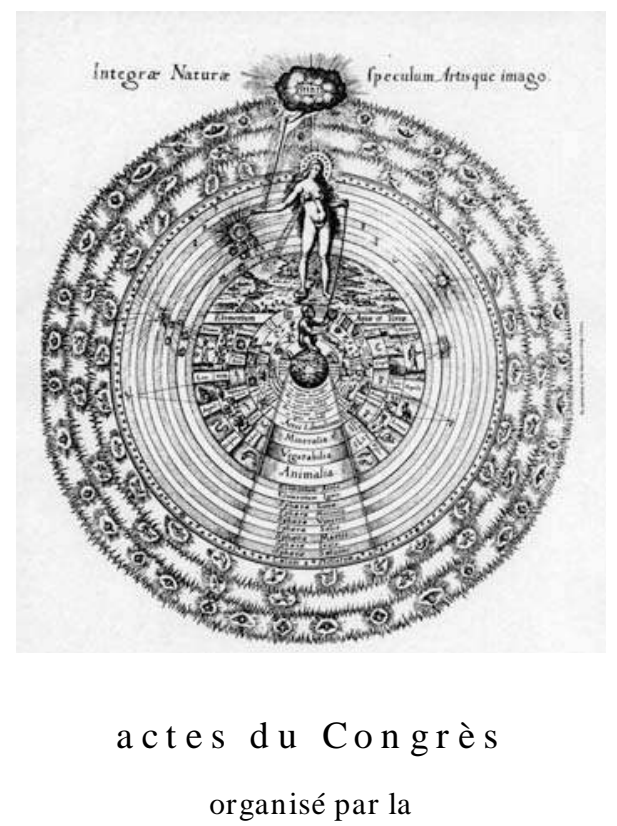

SOCIÉTÉ FR ANÇAISE SH AKESPEARE en collaboration avec la

S OC I É T É I N T E R N A T I O N A L E DES AM I D E M O N A I G N les 13,14 et 15 mars 2003

$$
\begin{gathered}
\text { textes réunis par } \\
\text { Pierre KAPITANIAK } \\
\text { sous la direction de } \\
\text { Jean-Marie MAGUIN }
\end{gathered}
$$




\section{COMITÉ SCIENTIFIQUE :}

Margaret Jones-Davis

Gisèle Venet

Jean-Marie Maguin

Yves Peyré

François Laroque

Pierre Kapitaniak

\section{COUVERTURE :}

Robert Fludd

Utriusque Cosmi Historia (1617-19)

planche 17

conception graphique et logo

Pierre Kapitaniak

\section{(C) 2003 Société Française Shakespeare}

Institut du Monde Anglophone

Université de Paris III - Sorbonne Nouvelle

http:// univ-montp3.fr/SFS/

5 rue de l'École de Médecine

75006 Paris

Diffusion :

AVL DIFFUSION

Parc Euromédecine

34198 MONTPELLIER CEDEX 5

ISBN 2-9521475-0-7

Tous droits de traduction, de reproduction et d'adaptation réservés pour tous les pays 


\title{
Plutarque Chez Montaigne ET CHEZ SHAKESPEARE
}

\author{
Gisèle MATHIEU-CASTELLANI
}

\begin{abstract}
Une forme de proximité entre Shakespeare et Montaigne a déjà été repérée, en particulier par l'autre géant, Hugo. On voudrait s'intéresser ici à la présence, chez l'un et chez l'autre, de Plutarque. Mais de quel Plutarque, au juste ? Chez Montaigne, Plutarque est surtout le modèle d'un psycho-historien, mais pas seulement cela... Chez Shakespeare, Plutarque est surtout un metteur en scène, un dramaturge virtuel, mais pas seulement cela... Chez l'un et l'autre, l'accent est mis décisivement sur les obscurités du sujet, son trouble, son inconsistance, son altérité. N'est-ce pas là l'héritage de Plutarque, du Plutarque auteur de l'E de Delphes? Si l'héritage est légué en voie directe à Montaigne, peut-être est-il transmis à Shakespeare par l'intermédiaire des Essais?

Plutarch in Montaigne and Shakespeare One sort of proximity between Shakespeare and Montaigne has already been noted by that other literary giant, Hugo. In this paper we will consider the presence of Plutarch in both writers. But which Plutarch do we mean, exactly? In Montaigne, Plutarch is above all the model of the psycho-historian, but not only that... In Shakespeare, he is above all a director, a virtual playwright, but not only that... In both, the emphasis is decisively placed on the dark areas of the subject, his turmoil, his inconsistency, his otherness. Is this not the heritage of Plutarch, the Plutarch who wrote the E of Delphes? Where the heritage is directly received by Montaigne, it is arguably passed on to Shakepeare through the Essais .
\end{abstract}

$\mathrm{D}$

'entrée de jeu, je dois avouer que si la confrontation proposée excitait la curiosité, elle me semblait d'abord risquée, tant les univers imaginaires de l'essayiste et du dramaturge me paraissent différents à bien des égards. Et pourtant... Et pourtant je sais bien qu'on a déjà mis en lumière l'importance des Essais dans la pensée de Shakespeare, au moins depuis Hugo :

Shakespeare pense, Shakespeare songe, Shakespeare doute. Il y a en lui de ce Montaigne qu'il aimait. Le To be or not to be sort du Que sais$j e^{287}$ ?

Dans sa précieuse notice d'Antoine et Cléopâtre, Gisèle Venet a mis en évidence plusieurs motifs qui rapprochent en effet la vision du monde de Shakespeare de celle de Montaigne ${ }^{288}$. Voici quelques-uns de ceux qu'elle a observés : l'imaginaire du mouvement, l'attention portée aux métamorphoses - et l'on sait que l'un et l'autre sont des lecteurs

287 V. Hugo, William Shakespeare, in Euvres Complètes, éd. J. Massin, Le Club français du livre, t. XII, 1969, p. 298

288 Dans la toute récente édition d'Antoine et Cléopâtre dans la Bibliothèque de la Pléiade (Paris, Gallimard, 2002), Gisèle Venet a rédigé une importante notice (p. 1488-1524), et les annotations en collaboration avec Line Cottegnies. Je remercie vivement Gisèle Venet, qui m’a communiqué la notice et les notes avant leur publication. 
attentifs d'Ovide -, «la soumission aux mouvements du temps »; mais aussi le refus du dogmatisme, et le goût pour l'ouverture qui laisse dans l'indécision le commentaire des actions, et le jugement porté sur les hommes. Ce n'est pas rien!

Mais c'est par un biais, et d'une vue oblique, que je m'essaierai ici au parallèle. Comme on sait que chez l'un et chez l'autre Plutarque plus précisément le Plutarque des Vies dans la traduction d'Amyot, traduite en anglais par North - affirme sa présence, je poserai la question ainsi : quel Plutarque au juste ? Et quel rôle joue-t-il ici et là ?

À première vue, des différences apparaissent : le Plutarque de Shakespeare semble être seulement celui des Vies, alors que Montaigne emprunte aussi aux Moralia, et doit en particulier beaucoup au traité De l'E de Delphes, traduit aussi par Amyot, «Que signifiait $e i »$; mais surtout si l'essayiste, qui apprécie dans les Vies les séquences comparatives où se pèsent les âmes des héros mis en parallèle, voit d'abord en Plutarque un psycho-historien, le dramaturge semble surtout voir en lui... un dramaturge, un premier Shakespeare, si l'on ose dire, le metteur en scène de drames flamboyants où la vie privée est étroitement liée à la vie publique, où les conflits personnels se trouvent intimement mêlés aux conflits sociaux et politiques, où le monde - le monde politique et social - est un théâtre, et ses acteurs des comédiens.

Néanmoins, on peut faire l'hypothèse que l'un et l'autre ont été séduits par tout ce que révèlent les analyses de Plutarque sur les ambiguités et les contradictions qui se font jour dans l'homme, et sur le caractère dramatique de l'existence: je dis dramatique, et non tragique $^{289}$, car la question se pose de savoir si cet être confus et obscur à lui-même qu'est le sujet des Essais, le héros des drames de Shakespeare, est affronté ici et là à une transcendance, à la fois audessus de sa personne et intériorisée...

Plutarque est en effet d'abord pour les écrivains de la Renaissance ce psycho-historien qui fait du genre classique de la Vie, la biographie des

289 Comme l'a fort bien montré Henri Gouhier dans Le théâtre et l'existence, Paris, Aubier, Montaigne, 1952, «il y a tragédie par la présence d'une transcendance, quelle que soit cette transcendance», mais il n'y a «point de transcendance qui ne soit corrélative d'une certaine immanence » (p. 42 et p. 41) 
hommes illustres, une autre forme d'histoire, distincte de ce qu'Amyot appelle l'histoire commune :

Or est-il, que selon la diversité de la matière qu'elle [l'histoire] traite, ou de l'ordre et manière d'écrire dont elle use, on lui donne noms différents : mais il y en a entre autres deux principales espèces : l'une qui expose au long les faits et aventures des hommes, s'appelle du nom commun d'histoire: l'autre qui déclare leur nature, leurs dits et leurs mœurs, qui proprement se nomme Vie. Et combien que leurs sujets soient fort conjoints, si est-ce que l'une regarde plus les choses, l'autre les personnes: l'une est plus publique, l'autre plus domestique: l'une concerne plus ce qui est au dehors de l'homme, l'autre ce qui procède du dedans : l'une les événements, et l'autre les conseils. (Aux Lecteurs) ${ }^{290}$

D’une certaine façon, cette analyse éclaire à la fois la matière et la manière de l'essai, et l'écriture de l'histoire dans le drame.

C'est bien en effet cette forme d'histoire appelée Vie qui est à l'horizon du genre de l'essai; celui-ci, s'il se présente volontiers comme la peinture d'une vie:

J'ai choisi le moment où ma vie, que j'ai à peindre, je l'ai toute devant $\operatorname{moi}^{291} \ldots$

n'est pas seulement ou pas d'abord cela : il entend surtout déclarer la nature, les dits et les mœurs de ce héros qu'est le scripteur; et écrire une histoire domestique, l'histoire d'un particulier dont la vie est basse et sans lustre :

Je propose une vie basse et sans lustre, c'est tout un. On attache aussi bien toute la philosophie morale à une vie populaire et privée qu'à une vie de plus riche étoffe...(III, 2, 805)

Une histoire qui concerne en effet « ce qui procède du dedans », non la geste d'un héros, mais l'existence d'un homme en lequel se voit, comme en chaque homme, la forme entière de l'humaine condition :

Les auteurs se communiquent au peuple par quelque marque particulière et étrangère; moi le premier par mon être universel,

290 Les Vies des hommes illustres de Plutarque, traduites du grec par Amyot, éd. Brotier et Vauvilliers, nouvelle éd. Clavier, tome premier, Paris, Imprimerie de Cussac, an IX (1801), «Aux Lecteurs », p. xlvii.

${ }^{291}$ Montaigne, Les Essais, éd. Villey-Saulnier, Paris, PUF, 1965, III, 12, p. 1057 ; toutes références selon cette édition (je modernise l'orthographe). 
comme Michel de Montaigne, non comme grammairien ou poète ou jurisconsulte. (ibid.)

Mon être universel: tout ce qui fait que je suis moi, « un particulier», un individu qui ne se réduit pas à son rôle social, mais se donne à voir dans son intimité, «tout entier et tout nu ». L'essai cependant présente cette notable différence avec la Vie que l'historien se confond ici avec le personnage présenté, et que l'écriture est d'abord écriture du moi. Moins autobiographie, certes, qu'autographie.

Ce que j'ai analysé ailleurs ${ }^{292}$ comme «les dépouilles de Plutarque » dans les Essais - pour reprendre la métaphore chère à Montaigne, présentant son livre comme «maçonné purement [des] dépouilles » de Sénèque et de Plutarque (II, 32, p. 721) - pourrait se résumer d'abord par l'interminable commentaire que l'essayiste fait de la préface d'Amyot, et sa réécriture. Il reprend ici par exemple - sans alléguer Amyot! - la féconde distinction entre les matières des deux sortes d'histoires, les conseils ici, les événements là, ici le dedans, là le dehors, en privilégiant évidemment la première forme :

Or ceux qui écrivent les vies, d'autant qu'ils s'amusent plus aux conseils qu'aux événements, plus à ce qui part du dedans qu'à ce qui arrive au dehors, ceux-là me sont plus propres. (II, 16, 416)

Et il justifie constamment la priorité accordée à l'auto-analyse d'un particulier, l'attention exclusive portée à ces mouvements qui procèdent « du dedans » :

Quel que soit donc le fruit que nous pouvons avoir de l'expérience, à peine servira beaucoup à notre institution celle que nous tirons des exemples étrangers, si nous faisons si mal notre profit de celle que nous avons de nous-même, qui nous est plus familière, et certes suffisante à nous instruire de ce qu'il nous faut. (III, 13, 1072)

Mais si Plutarque traduit et commenté par Amyot apporte une espèce de caution théorique au genre neuf de l'essai, à cette nouvelle forme d'histoire qui récuse la «narration étendue» des chroniqueurs et des historiens, on ajoutera aussi à l'héritage plusieurs legs : d'un côté les emprunts thématiques, les bribes narratives, les "contes", les exemples - et notamment les histoires de bêtes qui fondent dans

292 Montaigne. L'écriture de l'essai, Paris, PUF, 1988, p. 63-89. 
l'Apologie la démolition des prétentions humaines ${ }^{293}$ - ; de l'autre surtout - un trait caractéristique de l'ordre et manière d'écrire: les articles à pièces décousues et les digressions - Amyot du reste ajoute aux digressions de Plutarque ses propres digressions, marquées comme telles -, les variations :

Il est des ouvrages en Plutarque où il oublie son thème, où le propos de son argument ne se trouve que par incident, tout étouffé en matière étrangère: voyez ses allures au Démon de Socrate. O Dieu, que ces gaillardes escapades, que cette variation a de beauté...(III, 9, 994)

Et le goût pour les comparaisons, pour la pesée des âmes, la balance :

Mais qui considérera la vérité de la chose et les hommes en eux-mêmes, à quoi Plutarque a plus visé, et à balancer leurs mœurs, leurs naturels, leur suffisance que la fortune, je pense [...] que Cicéron et le vieux Caton en doivent de reste à leurs compagnons [...]. Mais c'est folie de vouloir juger d'un trait les choses à tant de visages. Quand Plutarque les compare, il ne les égale pas pourtant...(II, 22, 726-7)

Encore faut-il noter cette différence: l'essayiste se soucie surtout de peser sa propre âme et ses divers visages, ses mœurs, son naturel, sa suffisance, dans une interminable auto-analyse, d'observer les traits de son comportement, non seulement sa vision du monde, mais sa façon d'être au monde, son vivre, comme il dit.

La différence des genres laisse supposer que ce n'est pas tout à fait ce Plutarque-là que l'on retrouve dans les drames de Shakespeare, et pourtant ce n'est pas non plus tout à fait un autre...

Plusieurs pièces, on le sait, ont pour « sources", comme on dit, les Vies, La vie de Timon d'Athènes, Coriolan, Jules César, Antoine et Cléopâtre. C'est à cette dernière que je m'attacherai, puisque la présence de l'Antonius de Plutarque-Amyot y est, on le sait aussi, particulièrement remarquable: "Jamais plus que dans Antoine et Cléopâtre sans doute Shakespeare n'aura été aussi fidèle à la source qu'il s'est choisie, la «Vie d'Antoine» de Plutarque», dit Gisèle Venet ${ }^{294}$, et les modalités de l'emprunt méritent en effet un bref examen.

293 La source : le traité de Plutarque traduit par Amyot «Quels animaux sont les plus avisés ».

294 G. Venet, Notice, op. cit., p. 1488. Les annotations de G. Venet et de L. Cottegnies montrent fort précisément ces emprunts. 
Il faut d'abord observer que, pour tout ce qui concerne l'histoire, les événements, l'arrière-plan historique, Shakespeare semble s'inspirer aussi des Guerres Civiles d'Appien, qui, prenant le relais des narrations de Tite-Live qui nous sont parvenues en fragments, rapporte les divers épisodes des luttes entre les triumvirs, et le partage des diverses possessions. En revanche les portraits, les présentations des personnages, les discours, les scènes, les anecdotes: tout cela renvoie fort précisément à la Vie d'Antoine de PlutarqueAmyot. En somme pour Shakespeare aussi, Plutarque est moins historien que moraliste et psychologue, il est ce psycho-historien qui met en scène « ce qui procède du dedans ».

La présence de la Vie se manifeste d'abord dans les détails, et ce souci n'est pas le moins intéressant, car on voit combien pour l'historien comme pour le dramaturge l'homme se révèle moins sans doute dans ses actions publiques que dans les gestes de la vie quotidienne. Tandis que Montaigne n'emprunte à cette Vie qu'une dizaine de brèves références à Antoine, et deux à Cléopâtre, en particulier l'allusion aux Commourants :

Pourrait-on pas la rendre [la mort] encore voluptueuse, comme les commourants d'Antonius et de Cleopatra? (III, 9, 984),

Shakespeare multiplie les références. Sont rappelés, en dehors des épisodes historiques qui émaillent les guerres civiles et les relations compliquées des triumvirs, Octave, Lépide, Antoine, quelques épisodes de la vie privée des amants, et, par exemple, la mention des huit sangliers rôtis préparés par le cuisinier d'Antoine pour douze convives ; Mécène interroge Enorbabus :

Eight wild-boars roasted whole at a breakfast, and but twelve persons here ; is this true? (II.ii.185-6)

«Huit sangliers rôtis tout entiers pour un déjeuner, et seulement douze personnes à table : est-ce vrai ?» (trad. H. Thomas).

C'est vrai parce que Plutarque le dit...Et voici son plaisant récit :

J'ai autrefois ouï raconter à mon grand-père Lamprias, qu'un Philotas médecin natif de la ville d'Amphissa, lui contait comme en ce temps-là il était en Alexandrie [...], et que l'un des maîtres queux de la maison d'Antonius [...] le mena quant et lui [...] pour lui montrer le grand appareil et la somptuosité d'un seul souper. Quand il fut en la cuisine, il y vit une infinité de viandes, et entre autres huit sangliers tout entiers 
que l'on rôtissait, dont il fut fort ébahi, disant qu'il devait avoir grand nombre de gens à ce souper. Le cuisinier s'en prit à rire, et lui répondit qu'il n'y en avait pas beaucoup, ains environ douze seulement: mais qu'il fallait que tout ce qui était mis sur table, fût cuit et servi à son point, et Antonius voudra, peut-être, souper tout à cette heure, ou bien d'ici à un peu de temps, ou possible qu'il le diffèrera plus tard, pour ce qu'il aura beaucoup bu sur jour ${ }^{295}$...

De même les errances nocturnes d'Antoine et Cléopâtre dans Alexandrie, rappelées dès l'ouverture de la pièce par cette invitation d'Antoine à Cléopâtre :

and all alone,

To-night we'll wander through the streets [...]

Come, my queen,

renvoient au récit plutarquien :

Elle trouvait toujours quelque nouvelle volupté par laquelle elle tenait sous sa main, et maîtrisait Antonius, ne l'abandonnant jamais, et jamais ne le perdant de vue ne de jour ne de nuit : car elle jouait aux dés, elle buvait, elle chassait ordinairement avec lui, elle était toujours présente quand il prenait quelque exercice de la personne: quelquefois qu'il se déguisait en valet pour aller la nuit rôder par la ville [...], elle prenait l'accoutrement de quelque chambrière et s'en allait battre le pavé et courir avec lui. (p. 307-308)

Shakespeare a aimé chez Plutarque le portrait diversifié d'Antoine, son insouciance, si étonnante chez un personnage de haut rang affronté à de si terribles combats, son intempérance, ses insolences habituelles, ses railleries, «cette façon insolente de railler dont il usait ordinairement en se moquant et brocardant un chacun» (p. 299), ses « rencontres et brocards» qui aux yeux de la reine «sentaient leur soudard à pleine bouche» (p. 304), son mode de vie, une vie dissolue, si étrange pour un homme de cette sorte :

Il ne se plaisait point aux gen s de bien et d'honneur, à cause du reste de sa vie abandonnée et dissolue [...], ains était haï d'eux, pour autant qu'ils avaient en abomination les banquets et ivrogneries qu'il faisait ordinairement, à heure indue, ses dépenses excessives, et qu'il était ordinairement entre femmes folles, et puis le jour il dormait ou se promenait tout ivre pour digérer le vin qu'il avait trop pris la nuit. (p. 276)

295 Plutarque, Antonius, trad. Amyot, éd. cit., tome 8, an X (1802), p. 305-6; toutes références à cette édition (je modernise l'orthographe). 
Le héros, par exemple, manifeste son goût pour la pêche... Charmian rappelle ainsi à Cléopâtre la «farce » qu'elle lui joua, lorsque, pour se moquer de ses talents de pécheur, elle lui fit pécher un poisson séché :

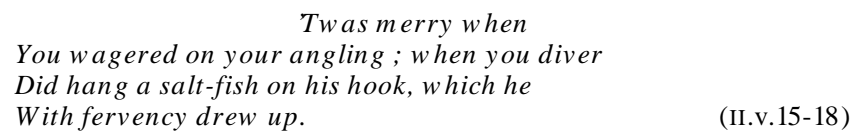

Plutarque se plaisait en effet à narrer cet épisode cocasse :

Il se mit quelquefois à pécher à la ligne, et voyant qu'il ne pouvait rien prendre, en était fort dépit et marri, à cause que Cleopatra était présente. Si commanda secrètement à quelques pécheurs, quand il aurait jeté sa ligne, qu'ils se plongeassent soudain en l'eau, et qu'ils allassent accrocher à son hameçon quelque poisson [...] Cleopatra s'en aperçut incontinent [...], mais à part elle conta le tour à ses familiers, et leur dit que le lendemain ils se trouvassent sur l'eau pour voir l'ébattement. Ils y vinrent sur le port en grand nombre, et se mirent dedans des bateaux de pécheurs, et Antonius aussi lâcha sa ligne, et lors Cleopatra commanda à l'un de ses serviteurs, qu'il se hâtât de plonger devant ceux d'Antonius, et qu'il allât attacher à l'hameçon de sa ligne quelque vieux poisson salé [...] et adonc, comme l'on peut penser, tous les assistants se prirent bien fort à rire, et Cleopatra en riant lui dit: «Laisse-nous, seigneur, à nous autres Egyptiens [...], laisse-nous la ligne : ce n'est pas ton métier : ta chasse est de prendre et conquérir villes et cités, pays et royaumes. » (p. 905)

Shakespeare s'intéresse lui aussi à Antoine lorsqu'il n'est pas ce triumvir occupé par ses luttes pour le pouvoir, "when he is not Antony», comme dit Philon dans la première scène. La fidélité au texte-source atteste que Shakespeare a découvert dans le personnage dessiné par Plutarque un héros shakespearien, sublime et grotesque, moqueur et moqué, inquiétant et ridicule, puissant et faible, courageux et lâche, un être double. Un homme dont les cheveux mêmes se querellent, s'accusant les uns les autres, les bruns de témérité, les blancs de crainte et d'imbécillité (III.xi).

Cette dualité interne du héros semble illustrer la méditation de Montaigne :

Nous sommes, je ne sais comment, doubles en nous-mêmes, qui fait que ce que nous croyons, nous ne le croyons pas, et ne nous pouvons défaire de ce que nous condamnons. (II, 16, 619) 
Je m'attacherai à quelques scènes hautes en couleurs, où l'on voit que le Plutarque dramaturge virtuel a inspiré son brillant héritier.

Voici d'abord la scène fascinante de la radieuse apparition de la reine sur le Cydnus. Chez Shakespeare la scène est prise dans un récit, la relation que fait Enobarbus à Mécène et à Agrippa :

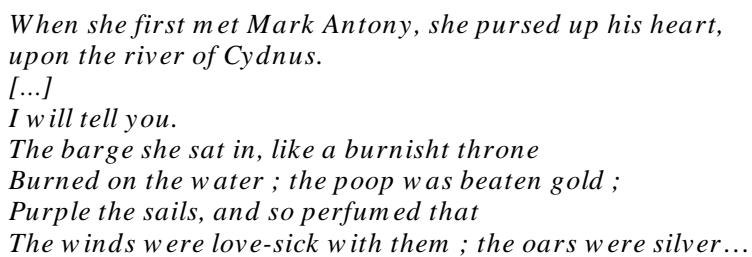

Le récit est d'une fidélité totale à Plutarque, qui présente ainsi la scène de la première rencontre :

\begin{abstract}
elle n'en daigna autrement s'avancer, sinon que de se mettre sur le fleuve Cydnus dedans un bateau, dont la poupe était d'or, les voiles de pourpre, les rames d'argent, que l'on maniait au son et à la cadence d'une musique de flûtes, hautbois, cythres, violes et autres tels instruments dont on jouait dedans. Et au reste, quant à sa personne, elle était couchée dessous un pavillon d'or tissu, vêtue et accoutrée toute en la sorte que l'on peint ordinairement Vénus, et auprès d'elle d'un côté et d'autre, de beaux petits enfants habillés ne plus ne moins que les peintres ont accoutumé de portraire les Amours, avec des éventaux en leurs mains, dont ils l'éventaient...(p. 302)
\end{abstract}

« La description, dit G. Venet, mobilise tous les sens, sollicite toute la subjectivité de l'imaginaire et non la seule objectivité du regard, comme déjà dans le texte de Plutarque auquel Shakespeare emprunte les couleurs chaudes, d'or et de pourpre, la musique des rames en cadence au son des flûtes, les jolis enfants potelés et leurs éventails...» (op. cit., p. 1503).

Et elle marque avec subtilité et précision la différence: une sensibilité à la menace, une érotisation des éléments, "l'image maniériste d'une sensation évanescente se défaisant en son contraire». Conservant l'ekphrasis, dit-elle, il en fait « un déni de représentation ».

Une autre scène attire l'attention : celle que jouent Antoine et Cléopâtre sur leurs chaires d'or. La description est à nouveau confiée à un tiers, César : 
I'th'market place, on a tribunal silvered Cleopatra and him self in chairs of gold Were publicy enthroned : at the feet sat Caesarion, whom they call my father's son, And all the unlawful issue that their lust

Chez Plutarque, l'initiative singulière d'Antoine est présentée comme un exemple caractéristique de son insolence et de sa superbe :

Car il fit assembler tout le peuple dedans le parc [...], et là, dessus un haut tribunal argenté fit mettre deux chaires d'or, l'une pour lui, et l'autre pour Cleopatra, et d'autres plus basses pour ses enfants; puis déclara publiquement devant toute l'assistance, qu'il établissait premièrement Cleopatra reine d'Égypte, de Cypre, de Lydie et de la basse Surie, et quant et elle Césarion aussi roi des mêmes royaumes.. (p. 351)

Ce qui fascine Shakespeare, c'est sans doute l'espèce de gratuité du geste insolent, l'excès dans l'ostentation, la provocation, la sorte de défi lancé à Fortune : Antonius est déjà un héros baroque, pour qui il n'y a point assez s'il n'y a trop... De Démétrius et d'Antoine Plutarque disait qu'ils étaient «insolents en leurs prospérités, et dissolus en voluptés et délices» (p.432); c'est bien ce trait qui retient l'attention de Shakespeare: héros irrationnel, Antoine se laisse conduire par son désir, même lorsque celui-ci contrarie ses projets « raisonnables» de conquérant.

Et voici enfin l'ultime scène, la plus saisissante, le célèbre suicide de la reine, se faisant apporter un aspic dans un panier, après avoir appris la mort de son amant. Chez Plutarque-Amyot, Cléopâtre, «outrée de tristesse et de mélancolie, et ensemble aussi de douleur» (p.421), s'enferme dans le tombeau d'Antoine pour offrir «les dernières oblations des morts» à son âme (p.426), puis met en oeuvre son suicide, «quand elle se fut baignée et lavée », au moment du repas :

Et cependant qu'elle dînait, il arriva un paysan des champs qui apportait un panier [...] ; il ouvrit son panier, et ôta les feuilles de figuier qui étaient dessus, et leur montra que c'étaient des figues. [...] Aucuns disent qu'on lui apporta l'aspic dedans ce panier avec les figues, et qu'elle l'avait ainsi commandé [...] Les autres disent qu'elle le gardait [l'aspic] dedans une buie, et qu'elle le provoqua et irrita avec un rameau d'or [...], mais il n'y a personne qui en sache rien à la vérité. (p. 425-7) 
Chez Shakespeare, dans l'ultime scène du drame, arrive un paysan porteur d'un panier plein de figues, et la reine l'interroge :

Hast thou the pretty worm of Nilus here,

That kills and pains not?

(V.ii.242-3)

« As-tu le gentil serpent du Nil, qui tue sans faire souffrir?»

Puis elle adresse ses adieux, et envoie un ultime message amoureux à celui qu'elle nomme son «époux » :

Husband, I come

Now to that name my courage prove my title!

I am fire and air..,

(V.ii.286-8)

comme chez Plutarque :

puis lui essuya le sang qui lui avait souillé la face, en l'appelant son seigneur, son mari et son empereur...(p. 416)

Tandis que résonnent pathétiquement ses graves dernières paroles :

As sweet as balm, as soft as air, as gentle-

O Antony! - Nay, I will take thee too

What should I stay -

(V.ii.310-2)

C'est en Plutarque le metteur en scène qui fascine le dramaturge, et un metteur en scène de goût baroque, qui mêle les genres, et le sublime au grotesque, le trivial au noble. Les bouffonneries du paysan chez Shakespeare «créent un instant de légèreté comique dans le moment le plus tragique », notent G. Venet et L. Cottegnies. Voici ensemble le rire et les larmes, la grossièreté et la noblesse des sentiments, le trivial au cœur même du tragique :

Shakespeare a la tragédie, la comédie, la féerie, l'hymne, la farce, le vaste rire divin, la terreur et l'horreur, et, pour tout dire en un mot, le drame ${ }^{296}$.

Le dramaturge a aimé cet Antoine de Plutarque qui aux habitants d'Alexandrie «montrait un visage comique, c'est-à-dire joyeux, et aux Romains un tragique, c'est-à-dire austère »(p.208), cet être partagé, intimement divisé.

296 V. Hugo, William Shakespeare, op. cit., p. 233. 
En effet, et sur ce point Montaigne et Shakespeare sont tout proches, Plutarque a apporté une vision des hommes qui avait tout pour les séduire. Au lieu d'une psychologie sommaire héritée de la rhétorique, d'une caractérologie soumise aux types, Shakespeare met en scène des êtres complexes, qui échappent à la définition, «toujours doubles en eux-mêmes", comme disait Montaigne. Et sur ce point il a bien entendu la leçon de Plutarque, qui présente le sujet comme «devenant toujours autre d'un autre ».

Antoine est-il seulement un ambitieux assoiffé de pouvoir? Certes, il fait tout ce qui lui est possible pour assurer sa domination, épousant, lors de son veuvage, non point la reine d'Égypte, mais la sœur de César, Octavia, et jouant auprès d'elle l'époux empressé. Mais il est aussi ce fou, «collé à Cléopâtre » comme dit Plutarque-Amyot, qui passe son temps en beuveries et en festins, et sacrifie sa nation à la passion que lui inspire une diablesse, celui qui a abandonné son empire à une catin, comme dit le César de Shakespeare (III.vi.66-67), celui qui a gaspillé pour des baisers des royaumes et des empires, selon Scarus (III.X). À la fois décidé et indécis, rusé et sincère, manipulateur et manipulé, il fait alterner déclarations enflammées d'amour et insultes pour la putain (whore), la triple putain, la catin, la goule (kite), la sorcière $($ spell $) \ldots$ Cléopâtre est du reste plus complexe chez Shakespeare que chez Plutarque-Amyot: à la fois putain et sainte, «transfigurée » au moment de sa mort héroïque, sans rien perdre de sa féminité troublante.

On se rappelle les belles pages de Hugo :

Qui dit poète dit en même temps et nécessairement historien et philosophe. [...] Shakespeare, lui aussi, est cet homme triple. Il est en outre le peintre, et quel peintre ! le peintre colossal ${ }^{297}$.

C'est alors le moment de se demander si en effet l'essayiste et le dramaturge n'ont pas l'un et l'autre entendu la même leçon de Plutarque, et pas seulement celle du psycho-historien, mais celle du philosophe.

Dans le traité Sur l'E de Delphes, Plutarque avait mis décisivement en lumière l'altérité du sujet, «genomenos heteros ex heterou», devenant toujours autre d'un autre, un être sans consistance, à qui la communication avec l'être est interdite.

297 Ibid., p. 233. 
Montaigne plagie - il n'est point d'autre mot pour cette réécriture textuelle, non seulement sans guillemets, mais sans aucune référence à l'auteur déplumé - une longue séquence du traité à la fin de son Apologie :

Nous n'avons aucune communication à l'être, parce que toute humaine nature est toujours au milieu entre le naître et le mourir [...]

Car il n'est pas vraisemblable que sans mutation nous prenions autres passions; et ce qui souffre mutation ne demeure pas un même, et, s'il n'est pas un même, il n'est donc pas aussi. Ainsi, quant et l'être tout un, change aussi l'être simplement, devenant toujours autre d'un autre. (II, 12, 601-603)

Voici la traduction d'Amyot :

Car à bien le prendre nous n'avons aucune participation du vrai être, parce que toute humaine nature est toujours au milieu, entre le naître et le mourir, ne baillant de soi qu'une obscure apparence et ombre, et une incertaine et débile opinion [...]

Car il n'est pas vraisemblable que sans mutation nous prenions autres passions, et ce qui souffre mutation ne demeure pas un même, et s'il n'est pas un même, il n'est donc pas aussi, ains quant et l'être tout un, change aussi l'être simplement, devenant toujours autre d'un autre ${ }^{298}$.

Je ne saurais dire si Shakespeare a lu aussi cette traduction d'Amyot, mais il a mis en scène somptueusement cette division intime du sujet, cette altérité qui interdit à l'homme d'être "un même», et qui fait, comme dit Montaigne, que «la ressemblance ne fait pas tant un comme la différence fait autre» (III, 13, 1065), et que «se trouve autant de différence de nous à nous-même que de nous à autrui. » (II, 1, 337). Telle est aussi, me semble-t-il, la pensée de Shakespeare : c'est bien cette différence inscrite au cœur du même qu'il scrute dans son héros.

C'est en effet ce qui est si étonnant chez Shakespeare et chez Montaigne : le caractère indécidable du «héros », quel qu'il soit. Et Plutarque en effet pratiquait avec art l'interrogation, s'interdisant de trancher dans le jugement porté sur les actions et les comportements, mettant en scène des héros dont la complexité est le trait commun, des héros toujours autres, sans cohérence. Chez lui déjà le peut-être, le sans doute, le probable, l'emportaient sur les certitudes et les assertion s catégoriques.

298 Euvres Mêlées de Plutarque, traduites du grec par Amyot, éd. cit., tome 19, Paris, Impr. de Cussac, an XI (1803), p. 51-53. 
Je dirais donc volontiers, pour conclure cet examen trop rapide, que la parenté observée entre ces deux géants par l'autre géant, Hugo, est à mettre en relation - en partie - avec leur commune généalogie : Plutarque, le Plutarque d'Amyot, a bien ensemencé nombre de pages de l'essayiste et du dramaturge, et ce Plutarque-là est certes le psychohistorien des Vies, mais aussi, sans doute, le philosophe moral qui, sachant que nous n'avons aucune communication à l'être, à l'essence, portait toute son attention sur l'existence, et ne cessait de scruter les ténèbres cimmériennes de la conscience, et l'étrange altérité inscrite au cœur du même.

Gisèle Mathieu-Castellani Université Paris-7 Denis-Diderot 